\title{
Modification of Chaos Game with Rotational Variation on a Square
}

\author{
Kosala Dwidja Purnomo', Indy Larasati², Ika Hesti Agustin ${ }^{3}$, Firdaus Ubaidillah4 \\ Department of Mathematics, University of Jember, Indonesia \\ Email: 1) kosala.fmipa@unej.ac.id, 2) indylarasati92@gmail.com, \\ 3) ikahesti.fmipa@unej.ac.id, 4) firdaus_u@yahoo.com
}

\begin{abstract}
Chaos game is a game of drawing a number of points in a geometric shape using certain rules that are repeated iteratively. Using those rules, a number of points generated and form some pattern. The original chaos game that apply to three vertices yields Sierpinski triangle pattern. Chaos game can be modified by varying a number of rules, such as compression ratio, vertices location, rotation, and many others. In previous studies, modification of chaos games rules have been made on triangles, pentagons, and n-facets. Modifications also made in the rule of random or nonrandom, vertex choosing, and so forth. In this paper we will discuss the chaos game of quadrilateral that are rotated by using an affine transformation with a predetermined compression ratio. Affine transformation is a transformation that uses a matrix to calculate the position of a new object. The compression ratio $r$ used here is 2 . It means that the distance of the formation point is $1 / 2$ of the fulcrum, that is $\alpha=1 / \mathrm{r}=1 / 2$. Variations of rotation on a square or a quadrilateral in chaos game are done by using several modifications to random and non-random rules with positive and negative angle variations. Finally, results of the formation points in chaos game will be analyzed whether they form a fractal object or not.
\end{abstract}

Keywords: chaos game, rotational variation, random, non-random.

\section{INTRODUCTION}

The original chaos game is a game of drawing a number of points on an equilateral triangle with certain rules that are repeated iteratively. Sierpinski triangle is an example of a fractal object formed by chaos game. Devaney [1] has modified the Sierpinski triangle with rotational variations that form fractal "films". The modification of Sierpinski triangle can be done by varying the angle of rotation, compression ratio, or location of the vertices for chaos game problems. Based on the research conducted by Devaney, this study will discuss the formation of chaos game on a square which are rotated by using an affine transformation with a certain compression ratio. The production rules are by using random and non-random rules, where random rules are built by selecting random points that can be modified with certain conditions, while non-random rules for point selection are carried out regularly and sequentially as many points as desired. The study was conducted to analyze whether the chaos game on a square varied by rotation with certain rules will still make fractals form such as Sierpinski triangle.

Fractals are defined as complex forms without limits because they have infinite details in a limited volume of space. This form will always look identical like an infinite pattern. However, if an object is made from elementary particles, then the details of the number of geometric shapes are limited. This amount is determined by the number of elementary particles and their relative position. The amount may be very large, but it is 
always limited [2]. Some examples of fractal forms include Cantor's middle third set, Von Koch curve, Sierpinski triangle, and Cantor dust [3].

Chaos game with three vertices is one method to generate Sierpinski triangle. The shape of vertices in chaos games can also be rectangles, pentagons, hexagons, and nfacets. Chaos games are generated using the specified compression ratio, namely the boundary between random points and vertices. The compression ratio is written with the symbol $r$ and the result is the new distance point $Z_{n+1}$ from the right point is $1 / r$ from the previous distance $Z_{n+1}$ to the vertex [4]. Chaos game is a point game where the production rules can still be developed, for example what if changing the number of vertices or changing the distance between the starting point and the specified vertex [5]. If the starting point in game chaos is located in a triangle, then the next marked point will always be located in the Sierpinski triangle. If the starting point is outside the triangle, then in some iteration a marked point will occur located in the triangle. Therefore, for some iteration the marked points are guaranteed to always be in the triangle [6].

Sierpinski triangle is a linear type of fractal that has identical self-similarity from one iteration to the next iteration. Sierpinski triangle is a triangle consisting of other triangles which are in the same shape but with half size. If a small part of the triangle is enlarged, it will look like another larger part. Affine transformation can generate Sierpinski triangle on chaos game with three vertices [7].

Transformation can be interpreted as a method that can be used to manipulate the location of a point. If the transformation is imposed on a set of points, then the object will change. Changes in this case are changes from the initial object location to the new location. The most commonly used method in graphical computer is the affine transformation method. An affine transformation is a transformation that uses a matrix to calculate the position of a new object. Transformation on two-dimensional objects can be in the form of translation, scale, and rotation.

Translation can be interpreted by changing locations from the initial location to the new location. The point $P(x, y)$ is shifted by a number of $\operatorname{Tr}_{x}$ on the $x$-axis and shifted by a number of $T r_{y}$ on the $y$-axis. Therefore the general formula will be obtained as follows

$$
Q(x, y)=P(x, y)+\operatorname{Tr}=P\left(x+\operatorname{Tr}_{x}, y+\operatorname{Tr}_{y}\right)
$$

Scale can be interpreted by an initial location multiplied by the desired quantity. The original location is multiplied by the amount $S_{x}$ on the $x$-axis and $S_{y}$ on the $y$-axis. So that the general formula will be obtained as follows

$$
Q(x, y)=P(x, y) \times S(x, y)=P\left(x \times S_{x}, y \times S_{y}\right) \text {. }
$$

Rotation can be interpreted by moving the initial location of an object (object) by rotating at an angle $\theta$ to the $x$-axis or $y$-axis. It is assuming that the centre point $(0,0)$ with a positive angle is counter-clockwise and a negative angle is in the direction clockwise. The rotation formula is as follows

$$
Q(x, y)=P(x, y) \times\left[\begin{array}{cc}
\cos \theta & -\sin \theta \\
\sin \theta & \cos \theta
\end{array}\right]
$$

Two-dimensional transformation uses two axes, namely the $x$-axis and the $y$-axis. The $x$-axis represents the length, while the $y$-axis represents the width [8]. We will modify the original chaos game on a square/quadrilateral by using geometric transformations, namely rotation, as Devaney done. The rules of affine transformation with linear algebraic principles for building a "fractal" film are formulated

$$
T\left(\begin{array}{l}
x \\
y
\end{array}\right)=\left(\begin{array}{cc}
1 / r & 0 \\
0 & 1 / r
\end{array}\right)\left(\begin{array}{cc}
\cos \theta & -\sin \theta \\
\sin \theta & \cos \theta
\end{array}\right)\left(\begin{array}{l}
x-x_{0} \\
y-y_{0}
\end{array}\right)+\left(\begin{array}{l}
x_{0} \\
y_{0}
\end{array}\right)
$$

The affine transformation combines the calculations of translation, scales and rotation 
matrices with a compression ratio written as $r$, and $\alpha$ is a distance that its value $1 / r$. The centre of rotation is $\left(x_{0}, y_{0}\right)$ with distance $\alpha$, and $\theta$ is the angle of rotation [9].

\section{RESULTS AND DISCUSSION}

Generation of modified chaos game on a square/quadrilateral will be done using random and non-random rules. The generation using random rules is done with some modifications. The compression ratio used is $r=2$, which means that the distance from the formation point is $1 / 2$ of the weight point (that is $\alpha=1 / \mathrm{r}$ ). There are four modifications here:

1. The last point that has been chosen cannot be chosen again;

2. The facing point cannot be chosen;

3. The left entry point cannot be chosen;

4. The right side point cannot be chosen.

The left and right points are seen from sequential patterns $A_{1}-A_{2}-A_{3}-A_{4}$, where the left point is the previous point, and the right point is the point after it. The exception is point $A_{3}$ where the left point is point $A_{4}$, then after choosing point $A_{3}$ it is not allowed to choose point $A_{4}$ and otherwise. Facing points also apply only to rectangles. The rules will be different if applied to pentagon and other aspects.

Non-random rules are also carried out with several modifications, namely nonrandom 1 with 4 regular and sequential point selection patterns $\left(A_{1}-A_{2}-A_{3}-A_{4}\right)$, nonrandom 2 with 16 point selection patterns, that is in multiples of 4 repeat from the last chosen point $\left(A_{1}-A_{2}-A_{3}-A_{3}-A_{4}-A_{1}-A_{2}-A_{2}-A_{3}-A_{4}-A_{1}-A_{1}-A_{2}-\right.$ $A_{3}-A_{4}-A_{4}$ ), non-random 3 with 20 point selection patterns, that is in multiples of 5 jumping over one point after that and in $t$ iterations namely $t \equiv 4 \bmod (5)$ the chosen point is the point facing the last point $\left(A_{1}-A_{2}-A_{3}-A_{1}-A_{3}-A_{4}-A_{1}-A_{2}-A_{4}-A_{2}-\right.$ $A_{3}-A_{4}-A_{1}-A_{3}-A_{1}-A_{2}-A_{3}-A_{4}-A_{2}-A_{4}$ ), non-random 4 with 24 point selection patterns, that is in multiples of 6 and in $t$ iterations namely $t \equiv 5 \bmod (6)$ repeats once like the previous point, then at $t \equiv 4 \bmod (6)$ jumps over one point after its last point $\left(A_{1}-\right.$ $A_{2}-A_{3}-A_{3}-A_{3}-A_{1}-A_{2}-A_{3}-A_{4}-A_{4}-A_{4}-A_{2}-A_{3}-A_{4}-A_{1}-A_{1}-A_{1}-A_{3}-$ $A_{4}-A_{1}-A_{2}-A_{2}-A_{2}-A_{4}$ ), and non-random 5 with 28 point selection patterns, in $t$ iterations namely $t \equiv 4 \bmod (7)$ jumps over one point from the last point, in the iteration $t \equiv 5 \bmod (7)$ repeats like the last point selected, at the iteration $t \equiv 6 \bmod (7)$ jumps over one point from the last point again, and in multiples of 7 repeats one point from the last point chosen $\left(A_{1}-A_{2}-A_{3}-A_{1}-A_{1}-A_{3}-A_{3}-A_{4}-A_{1}-A_{2}-A_{4}-A_{1}-A_{2}-A_{4}-A_{4}-\right.$ $\left.A_{2}-A_{2}-A_{3}-A_{4}-A_{1}-A_{3}-A_{3}-A_{1}-A_{1}-A_{2}-A_{3}-A_{4}-A_{2}-A_{2}-A_{4}-A_{4}\right)$.

Steps to make a chaos game:

a. Make a rectangle and give a name to each rectangular vertex, for example $A_{1}, A_{2}, A_{3}$, and $A_{4}$;

b. Choose a random starting point and is named $T_{0}$;

c. Select one vertex in the square to be associated with the starting point $T_{0}$ to make the game chaotic;

d. Determining the new point named $T_{1}$ and the distance $(\alpha)$ is half the distance of the vertex to the starting point according to the predetermined compression ratio, namely $r=2$;

e. Repeating steps (c) with the starting point is a new point obtained from the previous iteration and may be repeated by selecting the vertex as a reference point;

f. Do the desired number of points to iterations; 
The rotation rule used is by using an affine transformation. Suppose the rotation is done with the rotation angle given $30^{\circ}$ and the fulcrum is $A_{4}$. Then the new point formed will shift by $30^{\circ}$ towards the chosen fulcrum $A_{4}$. The difference in random and nonrandom game chaos formation lies in the selection of random points where points may be chosen randomly according to the desired modification rules, while the selection of points on non-random rules is determined by the desired pattern, in this study 4 patterns were selected, 16 patterns, 20 patterns, 24 patterns and 28 point repetition patterns.

The results of the forms obtained by random rules and non-random chaos games with positive and negative angle values will be compared, and the fulcrum of rotation is at the quadrilateral angle. The visualization results in chaos game on a square by random rules and their modification is done by rotation of angles every $10^{\circ}, \alpha=1 / 2$ and iterations of 10,000 . All objects generated in the rotation of positive angles are random rules and their modifications can be said to be fractal objects because they have similar shapes to each other (self-similarity). The rotation at the negative angle is also a fractal object. The difference is in the direction of rotation, where the rotation of the positive angle rotates counter-clockwise while the rotation of the negative angle rotates clockwise. The visualization for the positive angle are shown in Figure 1 to Figure 5.
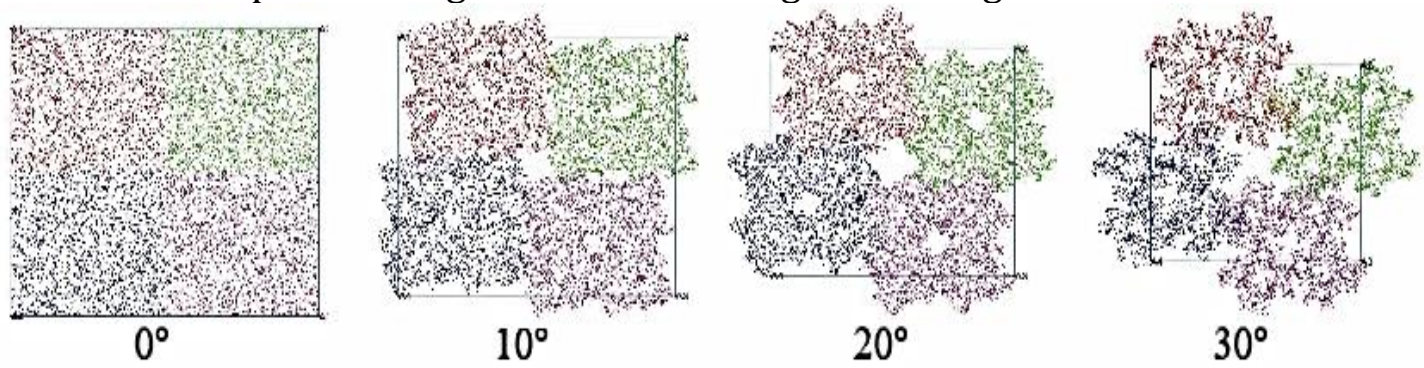

Figure 1. Rotational variation with random rules

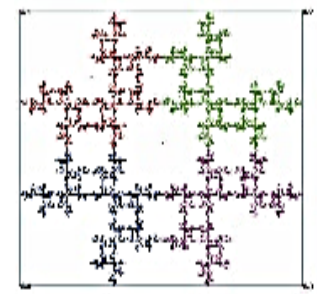

$0^{\circ}$

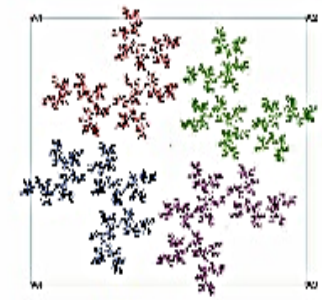

$10^{\circ}$

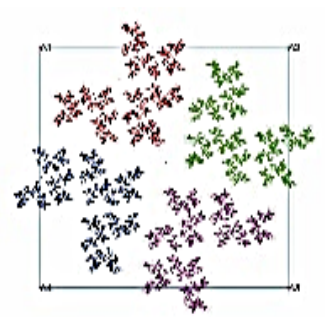

$20^{\circ}$

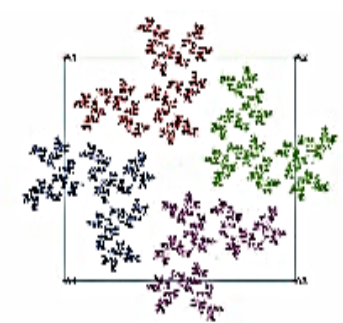

$30^{\circ}$

Figure 2. Rotational variation with modification 1

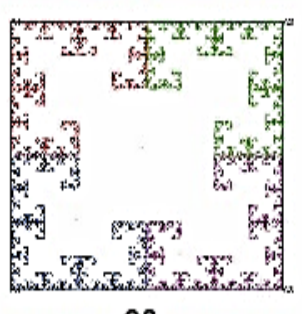

$0^{\circ}$

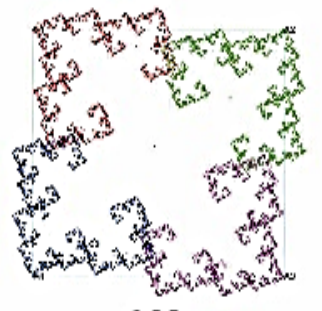

$10^{\circ}$

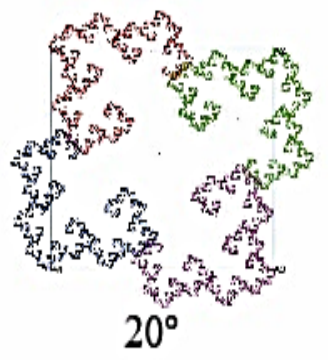

$20^{\circ}$

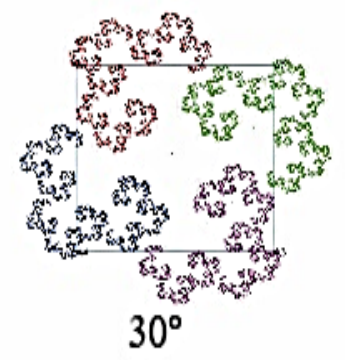

Figure 3. Rotational variation with modification 2 

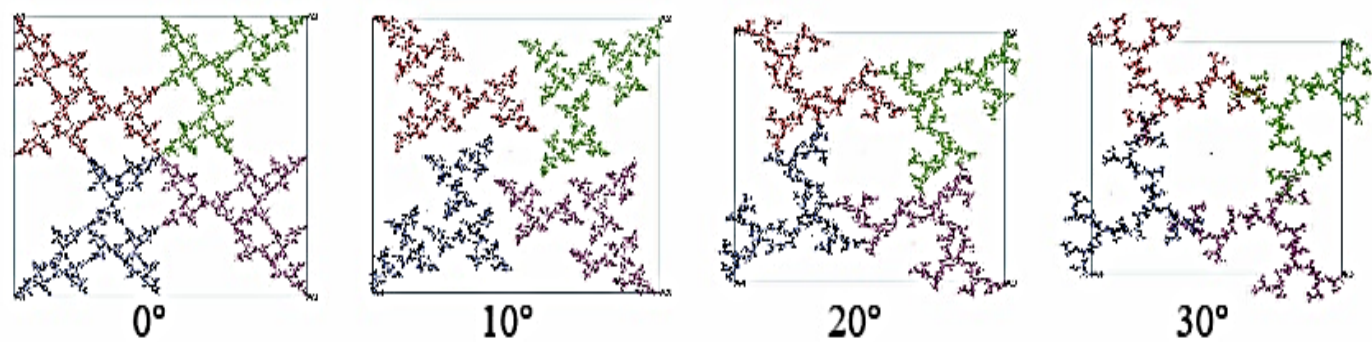

Figure 4. Rotational variation with modification 3
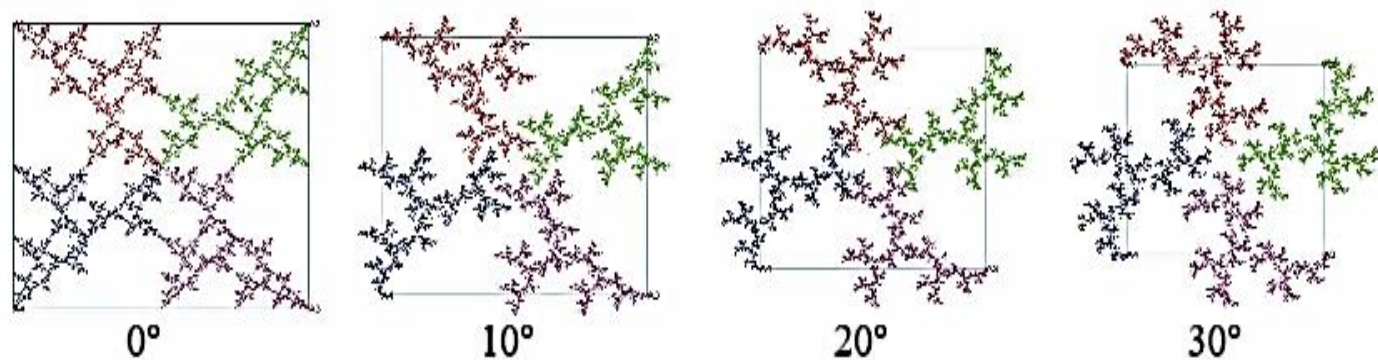

Figure 5. Rotational variation with modification 4

The visualization of the rules of non-random chaos games is done with $\alpha=1 / 2$, 5,000 iterations, and at positive angles of rotation every $30^{\circ}$ it does not produce a clear shape, but only like far apart points. The visualization of non-random rules simulation and their modifications shown in Figure 6 to Figure 7.

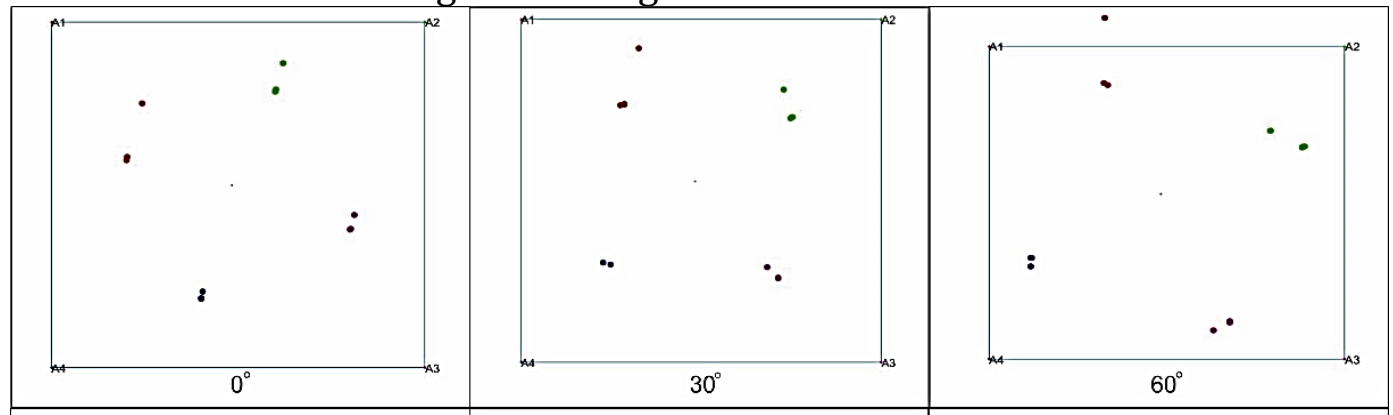

Figure 6. Rotational variation with non-random 1

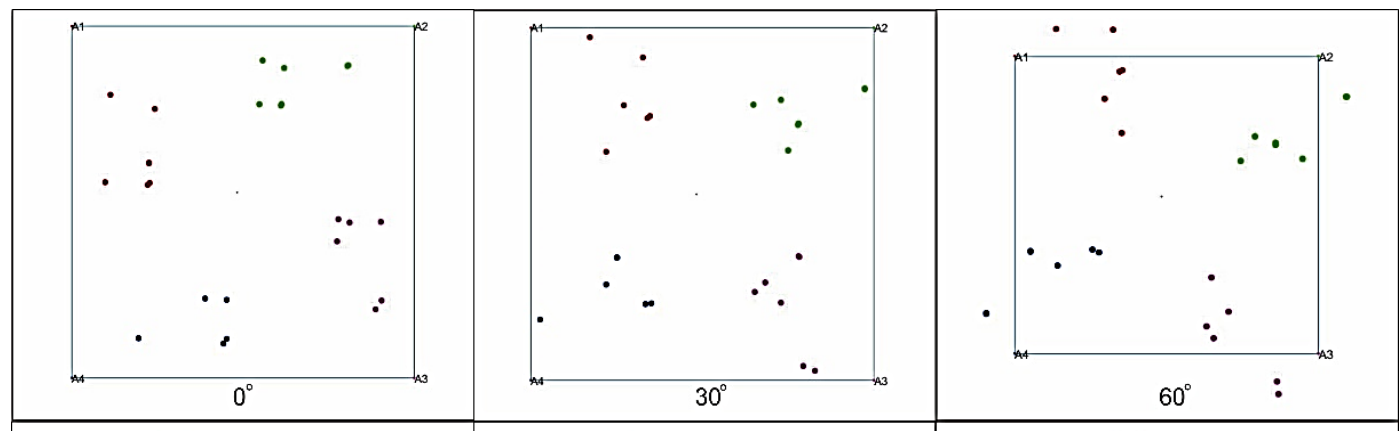

Figure 7. Rotational variation with non-random 2

The resulted points of non-random chaos game at a certain coordinate are due to the continuous selection of vertices that are patterned regularly. The points of the formation are spread unevenly and only "convergent" to a certain point as much as the pattern specified, and not converging on the four points of the support point as stated in previous studies. This causes the results of the chaos game with non-random rules not a 
fractals form because they have no similarity to each other (have no self-similarity). The coordinates of the new points generated from the last few iterations of non-random rules 1 to non-random rules 2 shown in Figure 8.

\begin{tabular}{|c|c|c|c|c|}
\hline \multicolumn{5}{|c|}{ Titik Baru } \\
\hline & pivot & $x$ & & $y$ \\
\hline 4900 & & & & \\
\hline 4981 & 1 & & 2 & 6 \\
\hline 4982 & 2 & & 6 & 8 \\
\hline 4983 & 3 & & 8 & 4 \\
\hline 4984 & 4 & & 4 & 2 \\
\hline 4985 & 1 & & 2 & 6 \\
\hline 4986 & 2 & & 6 & 8 \\
\hline 4987 & 3 & & 8 & 4 \\
\hline 4988 & 4 & & 4 & 2 \\
\hline 4989 & 1 & & 2 & 6 \\
\hline 4990 & 2 & & 6 & 8 \\
\hline 4991 & 3 & & 8 & 4 \\
\hline 4992 & 4 & & 4 & 2 \\
\hline 4993 & 1 & & 2 & 6 \\
\hline 4994 & 2 & & 6 & 8 \\
\hline 4995 & 3 & & 8 & 4 \\
\hline 4996 & 4 & & 4 & 2 \\
\hline 4997 & 1 & & 2 & 6 \\
\hline 4998 & 2 & & 6 & 8 \\
\hline 4999 & 3 & & 8 & 4 \\
\hline 5000 & 4 & & 4 & 2 \\
\hline
\end{tabular}

\begin{tabular}{|c|c|c|c|c|c|c|c|c|c|c|c|}
\hline \multicolumn{4}{|l|}{ Thik Baru } & \multicolumn{4}{|c|}{ Iitik Baru } & \multicolumn{4}{|c|}{ Thtlk Baru } \\
\hline 4340 & pivot & $x_{\text {rian }}$ & $\begin{array}{l}y \\
v_{v, m}\end{array}$ & 4700 & pivot & $x_{1 \text { ins }}^{x}$ & $\begin{array}{l}y \\
\text { risev }\end{array}$ & 4900 & pivot & $\begin{array}{l}x \\
\text { yerrov } \\
\end{array}$ & $y$ \\
\hline 4941 & 2 & 55642 & 90272 & 4961 & 1 & 0.9728 & 55642 & 4981 & 4 & 4.4358 & 0.9723 \\
\hline 4942 & 3 & 7.7821 & 45136 & 4962 & 2 & 54864 & 7.7821 & 4982 & 1 & 22179 & 54864 \\
\hline 4943 & 4 & 38911 & 22568 & 4963 & 3 & 7.7432 & 38911 & 4983 & 2 & 6.1069 & 7.7432 \\
\hline 4944 & 4 & 19455 & 11284 & 4964 & 3 & 8.8716 & 19455 & 4934 & 2 & 80545 & 88715 \\
\hline 4945 & 1 & 09728 & 5.5642 & 4965 & 4 & 4.4358 & 0.9728 & 4985 & 3 & 90272 & 4.4358 \\
\hline 4946 & 2 & 54864 & 7.7821 & 4966 & 1 & 22179 & 5.4864 & 4926 & 4 & 45136 & 22179 \\
\hline 4947 & 3 & 7.7432 & 38911 & 4967 & 2 & 6.1089 & 7.7432 & 4987 & 1 & 22568 & 6.1089 \\
\hline 4948 & 3 & 88716 & 1.2455 & 4968 & 2 & 8.0545 & 8.8716 & 4988 & 1 & 1.1204 & 80545 \\
\hline 4949 & 4 & 44358 & 0.9728 & 4969 & 3 & 90272 & 4.4358 & 4989 & 2 & $5 \leqslant 542$ & 90272 \\
\hline 4950 & 1 & 22179 & 54864 & 4970 & 4 & 4.5136 & 22179 & 4990 & 3 & 7.7821 & 4.5136 \\
\hline 4951 & 2 & 6.1009 & 7.7432 & 4971 & 1 & 2.2568 & 6.1089 & 4991 & 4 & 38911 & 2.2563 \\
\hline 4952 & 2 & 80545 & 8.8716 & 4972 & 1 & 1.1284 & 80545 & 4992 & 4 & 1.9155 & 1.1284 \\
\hline 4953 & 3 & 90272 & 44358 & 4973 & 2 & 55642 & 90272 & 4993 & 1 & 09728 & 55642 \\
\hline 4954 & 4 & 45135 & 22179 & 4974 & 3 & 7.7821 & 45136 & 4994 & 2 & 5.4364 & 7.7821 \\
\hline 4955 & 1 & 22568 & 6.1089 & 4975 & 4 & 3.8911 & 22568 & 4995 & 3 & 77432 & 38911 \\
\hline 4956 & 1 & 1.1284 & 80545 & 4976 & 4 & 19.455 & 1.1284 & 4996 & 3 & 8.8716 & 1.2855 \\
\hline 4957 & 2 & 55642 & 90272 & 4977 & 1 & 0.9728 & 5.5642 & 4997 & 4 & 44358 & 09723 \\
\hline 4958 & 3 & 7.7821 & 4.5136 & 4978 & 2 & 5.4864 & 7.7821 & 4998 & 1 & 22179 & 5.4864 \\
\hline 4959 & 4 & 38911 & 22568 & 4979 & 3 & 7.7432 & 3.8911 & 4999 & 2 & 6.1089 & 77432 \\
\hline 4960 & 4 & 19455 & 1.1284 & 4980 & 3 & 8.8716 & 19455 & 5000 & 2 & 80545 & 8.8716 \\
\hline
\end{tabular}

Figure 8. New coordinate points of non-random 1 (left) and non-random 2 (right)

It is different from random rules, where all the modifications form fractal objects because the selection of points is done randomly. According to [3] this is because the main principle of game chaos is to use chaos theory, which is a description of the complex movements and behavior of certain non-linear dynamic systems whose movements depend heavily on the initial state. Chaos formation of games with point selection is done non-randomly with a regular pattern that is determined as much as anything but still will not form a fractal due to the selection of regular and recurring points, so that the point will gather at a certain point. Previous studies that combined random and non-random rules also did not show fractal objects due to the involvement of non-random patterns, so they did not form fractals, although there were some patterns that were almost fractal, but less identical and still said that the object was not is a fractal.

\section{CONCLUSIONS}

Rotational variation of chaos game on a square/quadrilateral are built by random rules and all their modifications produce fractal objects because they have similar shapes (have self-similarity). Whereas the chaos game with non-random rules which the pattern is repeating points every 4 patterns, 16 patterns, 20 patterns, 24 patterns are not fractal objects because the formation points are far apart so they do not have similarity in form to one another points, but they have the characteristic of "convergent" repeating at certain coordinate points as much as the pattern of selection of a specified point.

Based on the results of the study, we get an open problem that can be done for further research:

1. Shows other modifications such as a combination of rotation variations and compression ratio;

2. Show analytic studies to prove that a chaos game object is a fractal 


\section{ACKNOWLEDGEMENTS}

We gratefully acknowledge the support from Keris Gerbang Mata and LP2M - University of Jember of year 2019.

\section{REFERENCES}

[1] Devaney, Robert L. 2003. Fractal Patterns and Chaos Game. Department of Mathematics: Boston University, Boston MA 02215.

[2] Shenker, O. R. 1994. Fractal Geometry is not the Geometry of Nature. Jerusalem: The Hebrew University of Jerusalem.

[3] Sulistiyantoko, D. 2008. Application of Sequence Row on Simple Fractal Geometry Calculation Formations. Essay. Mathematics Education Program, Faculty of Science and Technology, Sunan Kalijaga UIN Yogyakarta.

[4] Miller, C. 2011. Communicating Mathematics III: Z-Corp 650. http://www.maths.dur.ac.uk.

[5] Yunaning, F. 2018. Study of the Rules for Non-Random Chaos Games in the Triangle. Essay. Faculty of MIPA, University of Jember.

[6] Zohuri, B. 2015. Dimensional Analysis and Self-Similarity Methods for Engineers and Scientist. Switzerland: Springer International Publishing.

[7] Purnomo, Kosala D. 2016. Study of Sierpinski Triangle Establishment using Affine

] Transformation. Journal of Mathematics: Faculty of MIPA, Universty of Udayana, vol. VI No. 2:86-92.

[8] Sulastri. 2007. Build Transformation in Three-dimensional Space Using Visual Basic 6.0. Journal of DYNAMIC Information Technology Volume XII, No. 1: 88-100. Stikubank University Faculty of Information Technology.

[9] Sundbye, L. 1997. Chaos and Fractals on the TI Graphing Calculator. Department of Mathematical and Computer Sciences: Metropolitan State College of Denver. 